\title{
Strong Differential Subordinations Obtained with New Integral Operator Defined by Polylogarithm Function
}

\author{
K. AL-Shaqsi \\ Department of Information Technology, Nizwa College of Technology, Ministry of Manpower, P.O. Box 75, 612, Oman \\ Correspondence should be addressed to K. AL-Shaqsi; khalifa.alshaqsi@nct.edu.om
}

Received 9 December 2013; Accepted 20 February 2014; Published 6 April 2014

Academic Editor: Heinrich Begehr

Copyright ( 2014 K. AL-Shaqsi. This is an open access article distributed under the Creative Commons Attribution License, which permits unrestricted use, distribution, and reproduction in any medium, provided the original work is properly cited.

By using the polylogarithm function, a new integral operator is introduced. Strong differential subordination and superordination properties are determined for some families of univalent functions in the open unit disk which are associated with new integral operator by investigating appropriate classes of admissible functions. New strong differential sandwich-type results are also obtained.

\section{Introduction}

Let $\mathscr{H}$ denote the class of analytic function in the open unit disk $\mathbb{U}=\{z:|z|<1\}$. For a positive integer $n$ and $a \in \mathbb{C}$, let

$$
\mathscr{H}[n, k]=\left\{f \in \mathscr{H}: f(z)=a+a_{n} z^{n}+a_{n+1} z^{n+1}+\cdots\right\},
$$

and let $\mathscr{H}_{0} \equiv \mathscr{H}[0,1]$. We also denote by $\mathscr{A}$ the subclass of $\mathscr{H}[a, 1]$, with the usual normalization $f(0)=f^{\prime}(0)-1=0$.

Let $f(z)=z+\sum_{k=2}^{\infty} a_{k} z^{k}$ and $g(z)=z+\sum_{k=2}^{\infty} b_{k} z^{k}$ be formal Maclaurin series. Then, the Hadamard product or convolution of $f$ and $g$ is defined by the power series $(f *$ $g)(z)=f(z) * g(z)=z+\sum_{k=2}^{\infty} a_{k} b_{k} z^{k}$.

Let the functions $f$ and $F$ in $\mathscr{H}$; then we say that $f$ is subordinate to $F$ in $\mathbb{U}$, and write $f \prec F$, if there exists a Schwarz function $w$ in $\mathscr{A}$ with $|w(z)|<1$ and $w(0)=0$ such that $f(z)=F(w(z))$ in $\mathbb{U}$. Furthermore, if the function $F(z)$ is univalent in $\mathbb{U}$, then $f(z) \prec F(z)(z \in \mathbb{U}) \Leftrightarrow f(0)=F(0)$ and $f(\mathbb{U}) \subset F(\mathbb{U})(\operatorname{cf}[1-3])$.

Let $\Phi_{\delta}(a ; z)$ denote the well-known generalization of the Riemann zeta and polylogarithm functions, or simply the $\delta$ th order polylogarithm function, given by

$$
\Phi_{\delta}(c ; z)=\sum_{k=1}^{\infty} \frac{z^{k}}{(k+c)^{\delta}},
$$

where any term with $k+c=0$ is excluded; see Lerch [4] and also [5, Sections 1.10 and 1.12]. Using the definition of the Gamma function [5, page 27], a simply transformation produces the integral formula

$$
\Phi_{\delta}(c ; z)=\frac{1}{\Gamma(\delta)} \int_{0}^{1} z\left(\log \frac{1}{t}\right)^{\delta-1} \frac{t^{c}}{1-t z} d t,
$$

$$
\operatorname{Re} c>-1, \quad \operatorname{Re} \delta>1 \text {. }
$$

Note that $\Phi_{-1}(0 ; z)=z /(1-z)^{2}$ is Koebe function. For more details about polylogarithms in theory of univalent functions, see Ponnusamy and Sabapathy [6] and Ponnusamy [7].

Now, for $f \in A$, we defined the following integral operator:

$$
\begin{aligned}
\mathscr{J}_{c}^{\delta} f(z) & =(1+c)^{\delta} \Phi_{\delta}(c ; z) * f(z) \\
& =-\frac{(1+c)^{\delta}}{\Gamma(\delta)} \int_{0}^{1} t^{c-1}\left(\log \frac{1}{t}\right)^{\delta-1} f(t z) d t
\end{aligned}
$$

where $c>0, \delta>1$ and $z \in \mathbb{U}$.

We also note that the operator $\mathscr{I}_{c}^{p} f(z)$ defined by (4) can be expressed by the series expansion as follows:

$$
\mathscr{I}_{c}^{\delta} f(z)=z+\sum_{k=2}^{\infty}\left(\frac{1+c}{k+c}\right)^{\delta} a_{k} z^{k} .
$$

Obviously, we have, for $(\delta, \lambda \geq 0)$,

$$
\mathscr{I}_{c}^{\delta}\left(I_{c}^{\lambda} f(z)\right)=I_{c}^{\delta+\lambda} f(z) .
$$


Moreover, from (5), it follows that

$$
z\left(\mathscr{I}_{c}^{\delta+1} f(z)\right)^{\prime}=(c+1) \mathscr{I}_{c}^{\delta} f(z)-c \mathscr{I}_{c}^{\delta+1} f(z) .
$$

We note that,

(i) for $c=0$ and $\delta=n$ ( $n$ is any integer), the multiplier transformation $\mathscr{I}_{0}^{n} f(z)=I^{n} f(z)$ was studied by Flett [8] and Sălăgean [9];

(ii) for $c=0$ and $\delta=-n\left(n \in \mathbb{N}_{0}=\{0,1,2,3, \ldots\}\right)$, the differential operator $\mathscr{I}_{0}^{-n} f(z)=D^{n} f(z)$ was studied by Sălăgean [9];

(iii) for $c=1$ and $\delta=n$ ( $n$ is any integer), the operator $\mathscr{I}_{1}^{n} f(z)=\mathscr{I}^{n} f(z)$ was studied by Uralegaddi and Somanatha [10];

(iv) for $c=1$, the multiplier transformation $\mathscr{I}_{1}^{\delta} f(z)=$ $\mathscr{I}^{\delta} f(z)$ was studied by Jung et al. [11];

(v) for $c=a-1(a>0)$, the integral operator $\mathscr{I}_{a-1}^{\delta} f(z)=$ $\mathscr{I}_{a-1}^{\delta} f(z)$ was studied by Komatu [12].

To prove our results, we need the following definition and theorems considered by Antonino and Romaguera [13], Antonino [14], G. I. Oros and G. Oros [15], and Oros [16].

Definition 1 (see $[13]$ cf $[14,15])$. Let $H(z, \zeta)$ be analytic in $\mathbb{U} \times \overline{\mathbb{U}}$ and let $f(z)$ be analytic and univalent in $\mathbb{U}$. Then, the function $H(z, \zeta)$ is said to be strongly subordinate to $f(z)$, or $f(z)$ is said to be strongly superordinate to $H(z, \zeta)$, written as $H(z, \zeta) \prec \prec f(z)$, if, for $\zeta \in \overline{\mathbb{U}}, H(z, \zeta)$ as the function of $z$ is subordinate to $f(z)$. We note that $H(z, \zeta) \prec \prec f(z)$ if and only if $H(0, \zeta)=f(0)$ and $H(\mathbb{U}, \overline{\mathbb{U}}) \subset f(\mathbb{U})$.

Definition 2 ([15] cf [1]). Let $\phi: \mathbb{C}^{3} \times \mathbb{U} \times \overline{\mathbb{U}} \rightarrow \mathbb{C}$ and let $h(z)$ be univalent in $\mathbb{U}$. If $p(z)$ is analytic in $\mathbb{U}$ and satisfies the (second-order) differential subordination

$$
\phi\left(p(z), z p^{\prime}(z), z p^{\prime \prime}(z) ; z, \zeta\right) \prec \prec h(z),
$$

then $p(z)$ is called a solution of the strong differential subordination. The univalent function $q(z)$ is called a dominant of the solution of the strong differential subordination, or more simply a dominant, if $p(z) \prec q(z)$ for all $p(z)$ satisfying (8). A dominant $\tilde{q}(z)$ that satisfies $\tilde{q} \prec q(z)$ for all dominants $q(z)$ of (8) is said to be best dominant.

Recently, Oros [16] introduced the following strong differential superordinations as the dual concept of strong differential subordination.

Definition 3 (see [16] cf [17]). Let $\varphi: \mathbb{C}^{3} \times \mathbb{U} \times \overline{\mathbb{U}} \rightarrow \mathbb{C}$ and let $h(z)$ be analytic in $\mathbb{U}$. If $p(z)$ and $\varphi\left(p(z), z p^{\prime}(z), z p^{\prime \prime}(z) ; z, \zeta\right)$ are univalent in $\mathbb{U}$ for $\zeta \in \bar{U}$ and satisfy the (second-order) strong differential superordination

$$
h(z) \prec \prec \varphi\left(p(z), z p^{\prime}(z), z p^{\prime \prime}(z) ; z, \zeta\right)
$$

then $p(z)$ is called a solution of the strong differential superordination. An analytic function $q(z)$ is called a subordinant of the solution of the strong differential superordination, or more simply a subordinant, if $q(z) \prec p(z)$ for all $p(z)$ satisfying (9). A univalent subordinant $\tilde{q}(z)$ that satisfies $q(z) \prec$ $\tilde{q}(z)$ for all subordinantes $q(z)$ of $(9)$ is said to be best subordinant.

Denote by $Q$ the class of function $q$ that are analytic and injective on $\overline{\mathbb{U}} \backslash E(q)$, where

$$
E(q)=\left\{\gamma \in \partial \mathbb{U}: \lim _{z \rightarrow \gamma} q(z)=\infty\right\},
$$

and such that $q^{\prime}(\gamma) \neq 0$ for $\gamma \in \overline{\mathbb{U}} \backslash E(q)$. Further, let the subclass of $\mathbb{Q}$ for which $q(0)=a$ be denoted by $Q(a)$ and Q(0) $\equiv Q_{0}$.

Definition 4 (see [15]). Let $\Omega$ be a set in $\mathbb{C}, q(z) \in \mathbb{Q}$ and let $n$ be a positive integer. The class of admissible functions $\Psi_{n}[\Omega, q]$ consists of those function $\psi: \mathbb{C}^{3} \times \overline{\mathbb{U}} \times \overline{\mathbb{U}} \rightarrow \mathbb{C}$ that satisfy the admissibility condition

$$
\psi(r, s, t ; z, \zeta) \notin \Omega,
$$

whenever $r=q(\gamma), s=k \gamma q^{\prime}(\gamma)$ and

$$
\operatorname{Re}\left\{\frac{t}{s}+1\right\} \geq k\left\{\frac{\gamma q^{\prime \prime}(\gamma)}{q^{\prime}(\gamma)}+1\right\}
$$

for $z \in \mathbb{U}, \gamma \in \partial \mathbb{U} \backslash E(q), \zeta \in \overline{\mathbb{U}}$, and $k \geq n$. We write $\Psi_{1}[\Omega, q]$ as $\Psi[\Omega, q]$.

Definition 5 (see [16]). Let $\Omega$ be a set in $\mathbb{C}$ and $q \in \mathscr{H}[a, n]$ with $q^{\prime}(z) \neq 0$. The class of admissible functions $\Psi_{n}^{\prime}[\Omega, q]$ consists of those function $\psi: \mathbb{C}^{3} \times \overline{\mathbb{U}} \times \overline{\mathbb{U}} \rightarrow \mathbb{C}$ that satisfy the admissibility condition

$$
\psi(r, s, t ; \gamma, \zeta) \in \Omega
$$

whenever $r=q(\gamma), s=z q^{\prime}(z) / m$ for $z \in \mathbb{U}$ and

$$
\operatorname{Re}\left\{\frac{t}{s}+1\right\} \leq \frac{1}{m}\left\{\frac{z q^{\prime \prime}(z)}{q^{\prime}(z)}+1\right\}
$$

for $z \in \mathbb{U}, \gamma \in \partial \mathbb{U}, \zeta \in \overline{\mathbb{U}}$, and $m \geq n \geq 1$. We write $\Psi_{1}^{\prime}[\Omega, q]$ as $\Psi^{\prime}[\Omega, q]$.

For the above two classes of admissible function, Oros and Oros proved the following theorems.

Theorem 6 (see [15]). Let $\psi \in \Psi_{n}[\Omega, q]$ with $q(0)=a$. If $p \in$ $\mathscr{H}[a, n]$ satisfies

$$
\psi\left(p(z), z p^{\prime}(z), z^{2} p^{\prime \prime}(z) ; z, \zeta\right) \in \Omega
$$

then $p(z) \prec q(z)$.

Theorem 7 (see [16]). Let $\psi \in \Psi_{n}^{\prime}[\Omega, q]$ with $q(0)=a$. If $p \in$ Q(a) and

$$
\psi\left(p(z), z p^{\prime}(z), z^{2} p^{\prime \prime}(z) ; z, \zeta\right)
$$


is univalent in $\mathbb{U}$ for $\zeta \in \bar{U}$, then

$$
\Omega \subset\left\{\psi\left(p(z), z p^{\prime}(z), z^{2} p^{\prime \prime}(z) ; z, \zeta\right): z \in \mathbb{U}, \zeta \in \bar{U}\right\}
$$

implies that $q(z) \prec p(z)$.

In the present paper, making use of polylogarithm function, we introduce a new integral operator. By using the differential subordination and superordination results given by G. I. Oros and G. Oros [15] and Oros [16], we determine certain classes of admissible functions and obtain some subordination and superordination implications of multivalent functions associated with the new integral operator $\mathscr{F}_{c}^{\delta}$ defined by (4). New differential sandwich-type theorems are also obtained. We remark that we use the same technique given by Cho [18].

\section{Subordination Results}

Firstly, we begin by proving the subordination theorem involving the integral operator $\mathscr{I}_{c}^{\delta}$ defined by (4). For this purpose, we need the following class of admissible functions.

Definition 8. Let $\Omega$ be a set in $\mathbb{C}, q \in Q_{0} \cap \mathscr{H}[0,1], \operatorname{Re}\{c\}>$ 0 , and $\delta \geq 1$. The class of admissible functions $\Phi_{\mathscr{F}}[\Omega, q]$ consists of those functions $\phi: \mathbb{C}^{3} \times \mathbb{U} \times \overline{\mathbb{U}} \rightarrow \mathbb{C}$ that satisfy the admissibility condition

$$
\phi(u, v, w ; z, \gamma) \notin \Omega,
$$

whenever

$$
\begin{gathered}
u=q(\zeta), \quad v=\frac{k \zeta q^{\prime}(\zeta)+c q(\zeta)}{c+1}, \\
\operatorname{Re}\left\{\frac{(c+1)^{2} w-c^{2} u}{(c+1) v-c u}-2 c\right\} \geq k, \quad \operatorname{Re}\left\{\frac{\zeta q^{\prime \prime}(\zeta)}{q^{\prime}(\zeta)}+1\right\}
\end{gathered}
$$

for $z \in \mathbb{U}, \zeta \in \partial \mathbb{U} \backslash E(q), \gamma \in \overline{\mathbb{U}}$, and $k \geq 1$.

Theorem 9. Let $\phi \in \Phi_{\mathscr{g}}[\Omega, q]$. If $f \in \mathscr{A}$ satisfies

$$
\begin{aligned}
& \left\{\phi\left(\mathscr{I}_{c}^{\delta+1} f(z), \mathscr{I}_{c}^{\delta} f(z), \mathscr{J}_{c}^{\delta-1} f(z) ; z, \gamma\right): z \in \mathbb{U}, \gamma \in \overline{\mathbb{U}}\right\} \\
& \subset \Omega
\end{aligned}
$$

then

$$
\mathscr{I}_{c}^{\delta+1} f(z) \prec q(z) .
$$

Proof. Define the function $f(z)$ in $\mathbb{U}$ by

$$
p(z):=\mathscr{I}_{c}^{\delta+1} f(z) .
$$

From (22) with the relation (7), we get

$$
\mathscr{I}_{c}^{\delta} f(z)=\frac{z p^{\prime}(z)+c p(z)}{(c+1)} .
$$

Further computations show that

$$
\mathscr{J}_{c}^{\delta-1} f(z)=\frac{z^{2} p^{\prime \prime}(z)+(2 c+1) z p^{\prime}(z)+c^{2} p(z)}{(c+1)^{2}} .
$$

Define the transformation from $\mathbb{C}^{3}$ to $\mathbb{C}$ by

$$
u=r, \quad v=\frac{s+c r}{(c+1)}, \quad w=\frac{t+(2+c) s+c r}{(c+1)^{2}} .
$$

Let

$$
\begin{aligned}
\psi(r, s, t ; z, \gamma) & =\phi(u, v, w ; z, \gamma) \\
& =\phi\left(r, \frac{s+c r}{(c+1)}, \frac{t+(2 c+1) s+c^{2} r}{(c+1)^{2}} ; z, \gamma\right) .
\end{aligned}
$$

Using (22), (23), and (24), from (26), we obtain

$$
\begin{aligned}
\psi & \left(p(z), z p^{\prime}(z), z^{2} p^{\prime \prime}(z) ; z, \gamma\right) \\
& =\phi\left(\mathscr{I}_{c}^{\delta+1} f(z), \mathscr{I}_{c}^{\delta} f(z), \mathscr{J}_{c}^{\delta-1} f(z) ; z, \gamma\right) .
\end{aligned}
$$

Hence, (20) becomes

$$
\psi\left(p(z), z p^{\prime}(z), z^{2} p^{\prime \prime}(z) ; z, \gamma\right) \in \Omega .
$$

Note that

$$
\frac{t}{s}+1=\frac{(c+1)^{2} w-c^{2} u}{(c+1) v-c u}-2 c
$$

and so the admissibility condition for $\phi \in \Phi_{\mathscr{F}}[\Omega, q]$ is equivalent to the admissibility condition for $\psi \in \Psi[\Omega, q]$. Therefore, by Theorem $6, p \prec q$ or

$$
\mathscr{I}_{c}^{\delta+1} f(z) \prec q(z)
$$

which evidently completes the proof of Theorem 9 .

If $\Omega \neq \mathbb{C}$ is a simply connected domain, then $\omega=h(\mathbb{U})$ for some conformal mapping $h$ of $\mathbb{U}$ onto $\Omega$. In this case, the class $\Phi_{\mathscr{I}}[h(\mathbb{U}), q]$ is written as $\Phi_{\mathcal{I}}[h, q]$. The following result is an immediate consequence of Theorem 9.

Theorem 10. Let $\phi \in \Phi_{\mathscr{I}}[h, q]$. If $f \in \mathscr{A}$ satisfies

$$
\phi\left(\mathscr{I}_{c}^{\delta+1} f(z), \mathscr{I}_{c}^{\delta} f(z), \mathscr{I}_{c}^{\delta-1} f(z) ; z, \gamma\right) \prec \prec h(z),
$$

then

$$
\mathscr{I}_{c}^{\delta+1} f(z) \prec q(z) .
$$

Our next result is an extension of Theorem 9 to the case where the behavior of $q$ on $\partial \mathbb{U}$ is not known.

Corollary 11. Let $\Omega \subset \mathbb{C}$ and let $q$ be univalent in $\mathbb{U}$ with $q(0)=1$. Let $\phi \in \Phi_{\mathscr{I}}\left[\Omega, q_{\rho}\right]$ for some $\rho \in(0,1)$ where $q_{\rho}(z)=q(\rho z)$. If $f \in \mathscr{A}$ satisfies

$$
\phi\left(\mathscr{I}_{c}^{\delta+1} f(z), \mathscr{I}_{c}^{\delta} f(z), \mathscr{I}_{c}^{\delta-1} f(z) ; z, \gamma\right) \in \Omega
$$

then

$$
\mathscr{I}_{c}^{\delta+1} f(z) \prec q(z)
$$


Proof. Theorem 9 yields $\mathscr{I}_{c}^{\delta+1} f(z) \prec q_{\rho}(z)$. The result is now deduced from $q_{\rho}(z) \prec q(z)$.

Theorem 12. Let $h$ and $q$ be univalent in $\mathbb{U}$ with $q(0)=0$ and set $q_{\rho}(z)=q(\rho z)$ and $h_{\rho}(z)=h(\rho z)$. Let $\phi: \mathbb{C}^{3} \times \mathbb{U} \times \overline{\mathbb{U}} \rightarrow \mathbb{C}$ satisfy one of the following conditions:

(1) $\phi \in \Phi_{\mathscr{I}}\left[h, q_{\rho}\right]$, for some $\rho \in(0,1)$,

(2) there exists $\rho_{0} \in(0,1)$ such that $\phi \in \Phi_{\mathscr{I}}\left[h_{\rho}, q_{\rho}\right]$ for all $\rho \in\left(\rho_{0}, 1\right)$.

If $f \in \mathscr{A}$ satisfies (31), then

$$
\mathscr{F}_{c}^{\delta+1} f(z) \prec q(z)
$$

Proof. Using the same technique given in [3, Theorem $2.3 \mathrm{~d}]$.

Case 1. By applying Theorem 9 , we obtain $p \prec q_{\rho}$. Since $q_{\rho} \prec$ $q$, we deduce that $p \prec q$.

Case 2. If we let $q_{r} h o(z)=q(\rho z)$, then

$$
\begin{aligned}
\phi & \left(p_{\rho}(z), z p_{\rho}^{\prime}(z), z^{2} p_{\rho}^{\prime \prime}(z) ; z, \gamma\right) \\
& =\phi\left(p(\rho z), \rho z p^{\prime}(\rho z), \rho z^{2} p^{\prime}(\rho) ; z, \gamma\right) \in h_{\rho}(\mathbb{U}) .
\end{aligned}
$$

By using Theorem 9 and the comment associated with (20) with $z=\rho z$, we obtain $p_{\rho}(z) \prec q_{r} h o(z)$, for $p \in\left(\rho_{0}, 1\right)$. By letting $\rho \rightarrow 1^{-}$, we obtain $p \prec q$.

The next theorem yields the best dominant of the differential subordination.

Theorem 13. Let $h$ be univalent in $\mathbb{U}$ and let $\phi: \mathbb{C}^{3} \times \mathbb{U} \times \overline{\mathbb{U}} \rightarrow$ $\mathbb{C}$. Suppose that the differential equation

$$
\begin{aligned}
& \phi\left(q(z), \frac{z q^{\prime}(z)+c q(z)}{(c+1)},\right. \\
& \left.\quad \frac{z^{2} q^{\prime \prime}(z)+(2 c+1) z q^{\prime}(z)+c^{2} q(z)}{(c+1)^{2}} ; z, \gamma\right)
\end{aligned}
$$$$
=h(z)
$$

has a solution $q$ with $q(0)=0$ and satisfies one of the following conditions:

(1) $q \in \mathcal{Q}_{0}$ and $\phi \in \Phi_{\mathscr{I}}[h, q]$,

(2) $q$ is univalent in $\mathbb{U}$ and $\phi \in \Phi_{\mathcal{I}}\left[h, q_{\rho}\right]$, for some $\rho \in$ $(0,1)$

(3) $q$ is univalent in $\mathbb{U}$ and there exists $\rho_{0} \in(0,1)$ such that $\phi \in \Phi_{\mathscr{I}}\left[h_{\rho}, q_{\rho}\right]$ for all $\rho \in\left(\rho_{0}, 1\right)$.

If $f \in \mathscr{A}$ satisfies (31), and

$$
\phi\left(\mathscr{I}_{c}^{\delta+1} f(z), \mathscr{I}_{c}^{\delta} f(z), \mathscr{J}_{c}^{\delta-1} f(z) ; z, \gamma\right)
$$

is analytic in $\mathbb{U}$, then

$$
\mathscr{I}_{c}^{\delta+1} f(z) \prec q(z)
$$

and $q$ is the best dominant.

Proof. Using the same technique given in [3, Theorem 2.3e].

We deduce that $q$ is a dominant from Theorems 10 and 12 . Since $q$ satisfies (37), it is also a solution of (31) and therefore $q$ will be dominated by all dominants. Hence, $q$ is the best dominant.

In the particular case $q(z)=M z, M>0$, and, in view of Definition 8, the class of admissible function $\Phi_{\mathscr{I}}[\Omega, q]$, denoted by $\Phi_{\mathscr{I}}[\Omega, M]$, is described below.

Definition 14. Let $\Omega$ be a set in $\mathbb{C}, \operatorname{Re}\{c\}>0, \delta \geq 1$ and $M>0$. The class of admissible function $\Phi_{\mathscr{I}}[\Omega, M]$ consists of function $\phi: \mathbb{C}^{3} \times \mathbb{U} \times \overline{\mathbb{U}} \rightarrow \mathbb{C}$, such that

$$
\begin{aligned}
& \phi\left(M e^{i \theta}, \frac{(k+c) M e^{i \theta}}{(c+1)}, \frac{L+\left[(2 c+1) k+c^{2}\right] M e^{i \theta}}{(c+1)^{2}} ; z, \gamma\right) \\
& \notin \Omega,
\end{aligned}
$$

whenever $z \in \mathbb{U}, \gamma \in \overline{\mathbb{U}}$, and $\{\operatorname{Re}\}\left\{L e^{i \theta}\right\} \geq(k-1) k M, \theta \in \mathbb{R}$ and $k \geq 1$.

Corollary 15. Let $\phi \in \Phi_{\mathscr{I}}[\Omega, M]$. If $f \in \mathscr{A}$ satisfies

$$
\phi\left(\mathscr{I}_{c}^{\delta+1} f(z), \mathscr{I}_{c}^{\delta} f(z), \mathscr{I}_{c}^{\delta-1} f(z) ; z, \gamma\right) \in \Omega,
$$

then

$$
\mathscr{I}_{c}^{\delta+1} f(z) \prec M z
$$

In the special case $\Omega=q(\mathbb{U})=\{w:|w|<M\}$, the class $\Phi_{\mathscr{I}}[\Omega, M]$ is simply denoted by $\Phi_{\mathscr{I}}[M]$.

Corollary 16. Let $\phi \in \Phi_{\mathscr{J}}[M]$. If $f \in \mathscr{A}$ satisfies

$$
\left|\phi\left(\mathscr{I}_{c}^{\delta+1} f(z), \mathscr{I}_{c}^{\delta} f(z), \mathscr{I}_{c}^{\delta-1} f(z) ; z, \gamma\right)\right|<M,
$$

then

$$
\left|\mathscr{I}_{c}^{\delta+1} f(z)\right|<M
$$

Corollary 17. Let $c>1, M>0$ and let $C(\gamma)$ be an anlaytic function in $\overline{\mathbb{U}}$ with $\{\operatorname{Re}\}\{\zeta C(\gamma)\} \geq 0$ for $\zeta \in \partial \mathbb{U}$. If $f \in \mathscr{A}$ satisfies

$$
\begin{aligned}
& \left|(c+1)^{2} \mathscr{J}_{c}^{\delta-1} f(z)-(c+1) \mathscr{J}_{c}^{\delta} f(z)-c^{2} \mathscr{J}_{c}^{\delta+1} f(z)+C(\gamma)\right| \\
& \quad<c M
\end{aligned}
$$

then

$$
\left|\mathscr{I}_{c}^{\delta+1} f(z)\right|<M
$$


Proof. This follows from Corollary 15 by taking $\phi(u, v$, $w ; z, \gamma)=(c+1)^{2} w-(c+1) v-c^{2} u+C(\gamma)$ and $\Omega=h(\mathbb{U})$, where $h(z)=c M z$. To use Corollary 15, we need to show that $\phi \in \Phi_{\mathscr{g}}[\Omega, M]$; that is, the admissible condition (40) is satisfied. This follows since

$$
\begin{aligned}
& \mid \phi\left(M e^{i \theta}, \frac{(k+c) M e^{i \theta}}{(c+1)}, \frac{L+\left[(2 c+1) k+c^{2}\right] M e^{i \theta}}{(c+1)^{2}} ; z, \gamma\right) \mid \\
&= \mid L+\left[(2 c+1) k+c^{2}\right] M e^{i \theta}-(k+c) M e^{i \theta} \\
& \quad-c^{2} M e^{i \theta}+C(\gamma) \mid \\
&=\left|L+(2 k-1) c M e^{i \theta}+C(\gamma)\right| \\
& \geq(2 k-1) c M+\operatorname{Re}\left\{L e^{-i \theta}+\operatorname{Re}\left\{C(\gamma) e^{-i \theta}\right\}\right\} \\
& \geq(2 k-1) c M+k(k-1) M+\operatorname{Re}\left\{C(\gamma) e^{-i \theta}\right\} \\
& \geq c M
\end{aligned}
$$

for $z \in \mathbb{U}, \gamma \in \overline{\mathbb{U}}$, and $\operatorname{Re}\left\{L e^{-i \theta}\right\} \geq(k-1) k m, \theta \in \mathbb{R}$ and $k \geq 1$. Hence, by Corollary 15 , we deduce the required results.

\section{Superordination and Sandwich-Type Results}

The dual problem of differential subordination, that is, differential superordination of the new integral operator $\mathscr{I}_{c}^{\delta}$ defined by (4), is investigated in this section. For this purpose, the class of admissible functions is given in the following definition.

Definition 18. Let $\Omega$ be a set in $\mathbb{C}, q \in \mathscr{H}[0,1]$ with $q^{\prime}(z) \neq 0, \operatorname{Re}\{c\}>0$ and $\delta \geq 1$. The class of admissible functions $\Phi_{\mathscr{f}}^{\prime}[\Omega, q]$ consists of those functions $\phi: \mathbb{C}^{3} \times \mathbb{U} \times$ $\overline{\mathbb{U}} \rightarrow \mathbb{C}$ that satisfy the admissibility condition

$$
\phi(u, v, w ; z, \gamma) \in \Omega,
$$

whenever

$$
\begin{gathered}
u=q(z), \quad v=\frac{z q^{\prime}(\zeta) / m+c q(z)}{c+1}, \\
\operatorname{Re}\left\{\frac{(c+1)^{2} w-c^{2} u}{(c+1) v-c u}-2 c\right\} \leq \frac{1}{m}, \quad \operatorname{Re}\left\{\frac{z q^{\prime \prime}(z)}{q^{\prime}(z)}+1\right\}
\end{gathered}
$$

for $z \in \mathbb{U}, \zeta \in \partial \mathbb{U} \backslash E(q), \gamma \in \overline{\mathbb{U}}$, and $m \geq 1$.

Theorem 19. Let $\phi \in \Phi_{\mathscr{I}}^{\prime}[\Omega, q]$. If $f \in \mathscr{A}, \mathscr{I}_{c}^{\delta+1} \in Q_{0}$, and

$$
\phi\left(\mathscr{I}_{c}^{\delta+1} f(z), \mathscr{I}_{c}^{\delta} f(z), \mathscr{I}_{c}^{\delta-1} f(z) ; z, \gamma\right)
$$

is univalent in $\mathbb{U}$, then

$$
\begin{aligned}
\Omega \subset\left\{\phi\left(\mathscr{I}_{c}^{\delta+1} f(z), \mathscr{I}_{c}^{\delta} f(z), \mathscr{I}_{c}^{\delta-1} f(z) ; z, \gamma\right): z \in \mathbb{U},\right. \\
\gamma \in \overline{\mathbb{U}}\} .
\end{aligned}
$$

implies that

$$
q(z) \prec \mathscr{I}_{c}^{\delta+1} f(z)
$$

Proof. From (27) and (51), we have

$$
\Omega \subset\left\{\psi\left(p(z), z p^{\prime} f(z), z^{2} p^{\prime \prime}(z) ; z, \gamma\right): z \in \mathbb{U}, \gamma \in \overline{\mathbb{U}}\right\} .
$$

From (25), we see that the admissibility condition for $\phi \epsilon$ $\Phi_{\mathscr{F}}^{\prime}[\Omega, q]$ is equivalent to the admissibility condition for $\psi$ as given in Definition 2. Hence, $\psi \in \Psi_{\mathscr{F}}^{\prime}[\Omega, q]$ and, by Theorem $9, q \prec p$ or

$$
q(z) \prec \mathscr{I}_{c}^{\delta+1} f(z)
$$

which evidently completes the proof of Theorem 19.

If $\Omega \neq \mathbb{C}$ is a simply connected domain, then $\omega=h(\mathbb{U})$ for some conformal mapping $h$ of $\mathbb{U}$ onto $\Omega$. In this case, the class $\Phi_{\mathscr{J}}^{\prime}[h(\mathbb{U}), q]$ is written as $\Phi_{\mathscr{J}}^{\prime}[h, q]$. Proceeding similarly as in the previous section, the following result is an immediate consequence of Theorem 19.

Theorem 20. Let $q \in \mathscr{H}[0,1]$ and let $h$ be analytic in $\mathbb{U}$ and let $\phi \in \Phi_{\mathscr{g}}^{\prime}[h, q]$. If $f \in \mathscr{A}, \mathscr{I}_{c}^{\delta+1} \in \mathbb{Q}_{0}$ and

$$
\phi\left(\mathscr{I}_{c}^{\delta+1} f(z), \mathscr{I}_{c}^{\delta} f(z), \mathscr{I}_{c}^{\delta-1} f(z) ; z, \gamma\right)
$$

is univalent in $\mathbb{U}$, then

$$
h(z) \prec \prec \phi\left(\mathscr{I}_{c}^{\delta+1} f(z), \mathscr{I}_{c}^{\delta} f(z), \mathscr{I}_{c}^{\delta-1} f(z) ; z, \gamma\right)
$$

implies that

$$
q(z) \prec \mathscr{J}_{c}^{\delta+1} f(z) .
$$

Theorems 19 and 20 can only be used to obtain subordinantes of differential superordination of the form (51) or (56). The following theorem proves the existence of the best subordinant of (56) for certain $\phi$.

Theorem 21. Let $h$ be univalent in $\mathbb{U}$ and let $\phi: \mathbb{C}^{3} \times \mathbb{U} \times \overline{\mathbb{U}} \rightarrow$ C. Suppose that the differential equation

$$
\begin{aligned}
& \phi\left(q(z), \frac{z q^{\prime}(z)+c q(z)}{(c+1)},\right. \\
& \left.\frac{z^{2} q^{\prime \prime}(z)+(2 c+1) z q^{\prime}(z)+c^{2} q(z)}{(c+1)^{2}} ; z, \gamma\right) \\
& =h(z),
\end{aligned}
$$

has a solution $q \in \mathbb{Q}_{0}$. If $\phi \in \Phi_{\mathscr{F}}^{\prime}[h, q], f \in \mathscr{A}, \mathscr{I}_{c}^{\delta+1} \in \mathbb{Q}_{0}$, and

$$
\phi\left(\mathscr{I}_{c}^{\delta+1} f(z), \mathscr{I}_{c}^{\delta} f(z), \mathscr{J}_{c}^{\delta-1} f(z) ; z, \gamma\right)
$$

is univalent in $\mathbb{U}$, then

$$
h(z) \prec \prec \phi\left(\mathscr{I}_{c}^{\delta+1} f(z), \mathscr{I}_{c}^{\delta} f(z), \mathscr{I}_{c}^{\delta-1} f(z) ; z, \gamma\right)
$$


implies that

$$
q(z) \prec \mathscr{I}_{c}^{\delta+1} f(z)
$$

and $q$ is the best subordinant.

Proof. The proof is similar to that of Theorem 13 and so it is omitted.

Combining Theorems 10 and 20, we obtain the following sandwich-type theorem.

Theorem 22. Let $h_{1}$ and $q_{1}$ be analytic functions in $\mathbb{U}$ and let $h_{2}$ be analytic function in $\mathbb{U}, q_{2} \in \mathbb{Q}_{0}$ with $q_{1}(0)=q_{2}(0)=$ 0 and $\phi \in \Phi_{\mathscr{J}}\left[h_{2}, q_{2}\right] \cap \Phi_{\mathscr{J}}^{\prime}\left[h_{1}, q_{1}\right]$. If $f \in \mathscr{A}, \mathscr{I}_{c}^{\delta+1} \in$ $\mathscr{H}[0,1] \cap \mathcal{Q}_{0}$, and

$$
\phi\left(\mathscr{I}_{c}^{\delta+1} f(z), \mathscr{I}_{c}^{\delta} f(z), \mathscr{I}_{c}^{\delta-1} f(z) ; z, \gamma\right)
$$

is univalent in $\mathbb{U}$, then

$$
\begin{aligned}
& h_{1}(z) \\
& \prec \prec \phi\left(\mathscr{I}_{c}^{\delta+1} f(z), \mathscr{I}_{c}^{\delta} f(z), \mathscr{I}_{c}^{\delta-1} f(z) ; z, \gamma\right) \prec \prec h_{2}(z) .
\end{aligned}
$$

implies that

$$
q_{1}(z) \prec \mathscr{I}_{c}^{\delta+1} f(z) \prec q_{2}(z)
$$

\section{Conflict of Interests}

The author declares that there is no conflict of interests regarding the publication of this paper.

\section{Acknowledgment}

The work presented here was supported by Ministry of Manpower, Sultanate of Oman.

\section{References}

[1] S. S. Miller and P. T. Mocanu, "Differential subordinations and univalent functions," The Michigan Mathematical Journal, vol. 28, no. 2, pp. 157-172, 1981.

[2] S. S. Miller and P. T. Mocanu, "On some classes of firstorder differential subordinations," The Michigan Mathematical Journal, vol. 32, no. 2, pp. 185-195, 1985.

[3] S. S. Miller and P. T. Mocanu, Differential Subordination, Theory and Application, vol. 225, Marcel Dekker, New York, NY, USA, 2000.

[4] M. Lerch, "Note sur la fonction $K(w, x, s)=\sum_{k=0}^{\infty} e^{2 k \pi i x}$," Acta Mathematica, vol. 11, no. 1-4, pp. 19-24, 1887.

[5] H. Bateman, Higher Transcendental Functions, vol. 1 of Edited by: A. Erdelyi, W. Mangnus, F. Oberhettinger, F. G. Tricomi, McGraw-Hill, New York, NY, USA, 1953.

[6] S. Ponnusamy and S. Sabapathy, "Polylogarithms in the theory of univalent functions," Results in Mathematics, vol. 30, no. 1-2, pp. 136-150, 1996.
[7] S. Ponnusamy, "Inclusion theorems for convolution product of second order polylogarithms and functions with the derivative in a halfplane," The Rocky Mountain Journal of Mathematics, vol. 28, no. 2, pp. 695-733, 1998.

[8] T. M. Flett, "The dual of an inequality of Hardy and Littlewood and some related inequalities," Journal of Mathematical Analysis and Applications, vol. 38, pp. 746-765, 1972.

[9] G. S. Sălăgean, "Subclasses of univalent functions," in Complex Analysis-Fifth Romanian-Finnish Seminar, vol. 1013 of Lecture Notes in Mathematics, pp. 362-372, Springer, Berlin, Germany, 1983.

[10] B. A. Uralegaddi and C. Somanatha, "Certain classes of univalent functions," in Current Topics in Analytic Function Theory, H. M. Srivastava and S. Own, Eds., pp. 371-374, World Scientific, Singapore, 1992.

[11] I. B. Jung, Y. C. Kim, and H. M. Srivastava, "The Hardy space of analytic functions associated with certain one-parameter families of integral operators," Journal of Mathematical Analysis and Applications, vol. 176, no. 1, pp. 138-147, 1993.

[12] Y. Komatu, "On analytic prolongation of a family of operators," Mathematica, vol. 32, no. 2, pp. 141-145, 1990.

[13] J. A. Antonino and S. Romaguera, "Strong differential subordination to Briot-Bouquet differential equations," Journal of Differential Equations, vol. 114, no. 1, pp. 101-105, 1994.

[14] J. A. Antonino, "Strong differential subordination and applications to univalency conditions," Journal of the Korean Mathematical Society, vol. 43, no. 2, pp. 311-322, 2006.

[15] G. I. Oros and G. Oros, "Strong differential subordination," Turkish Journal of Mathematics, vol. 33, no. 3, pp. 249-257, 2009.

[16] G. Oros, "Strong differential superordination," Acta Universitatis Apulensis, vol. 19, pp. 101-106, 2009.

[17] S. S. Miller and P. T. Mocanu, "Subordinants of differential superordinations," Complex Variables. Theory and Application, vol. 48, no. 10, pp. 815-826, 2003.

[18] N. E. Cho, "Strong differential subordination properties for analytic functions involving the Komatu integral operator," Boundary Value Problems, vol. 2013, article 44, 2013. 


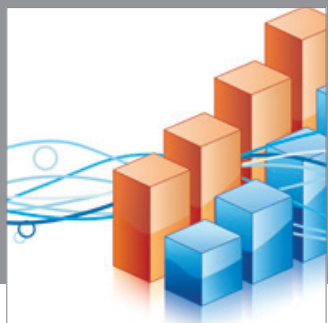

Advances in

Operations Research

mansans

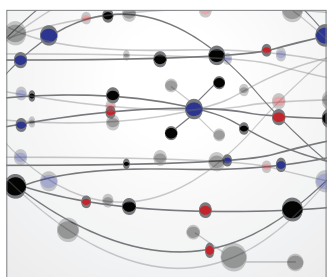

The Scientific World Journal
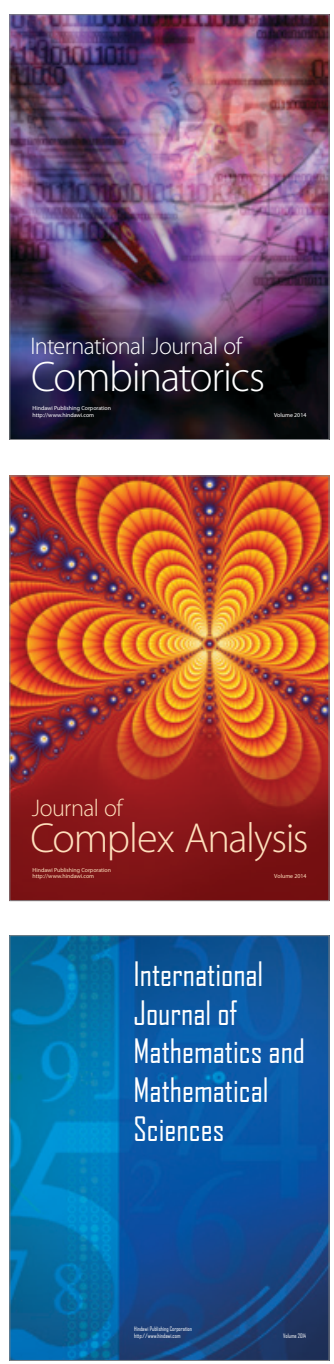
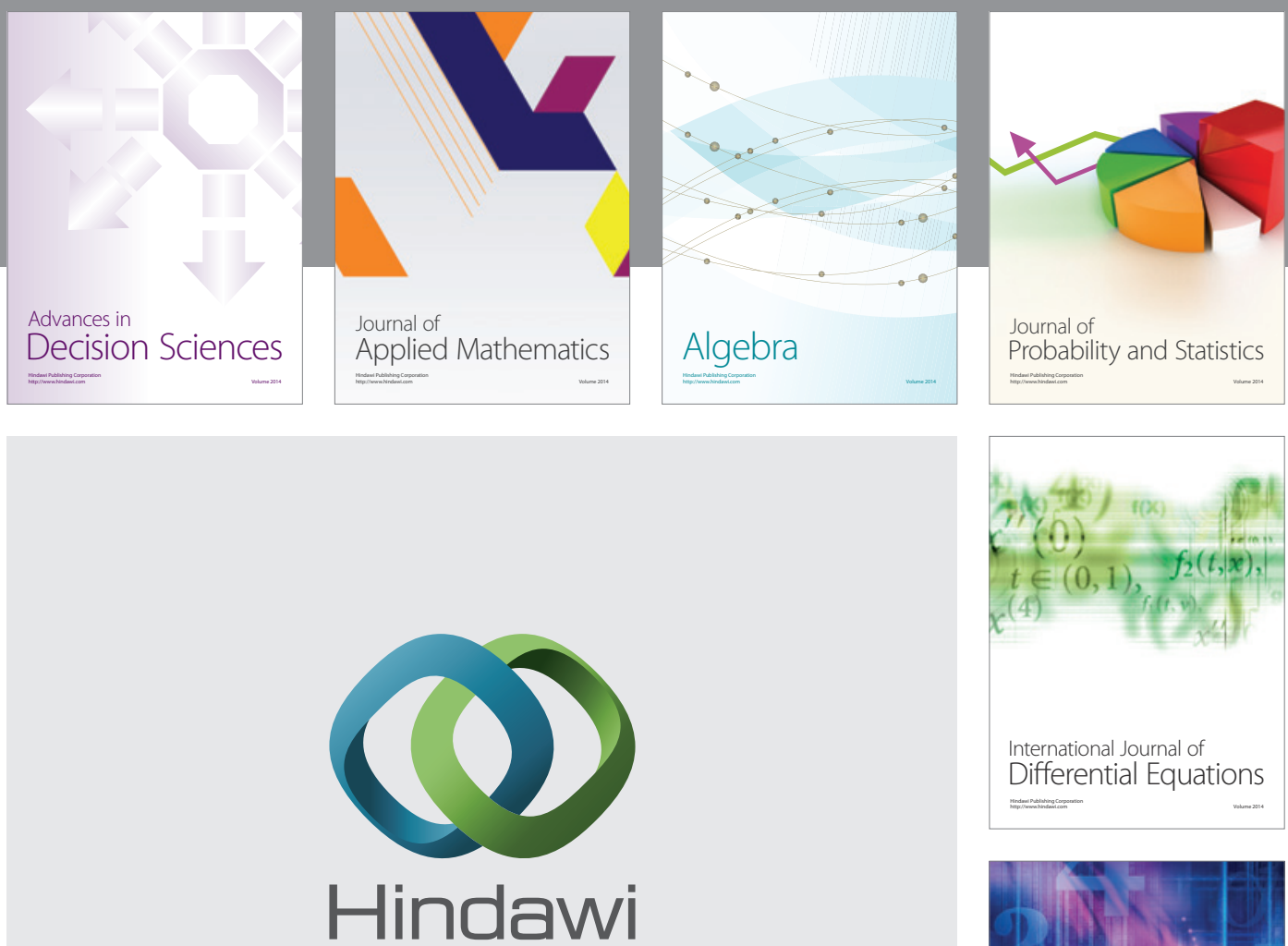

Submit your manuscripts at http://www.hindawi.com
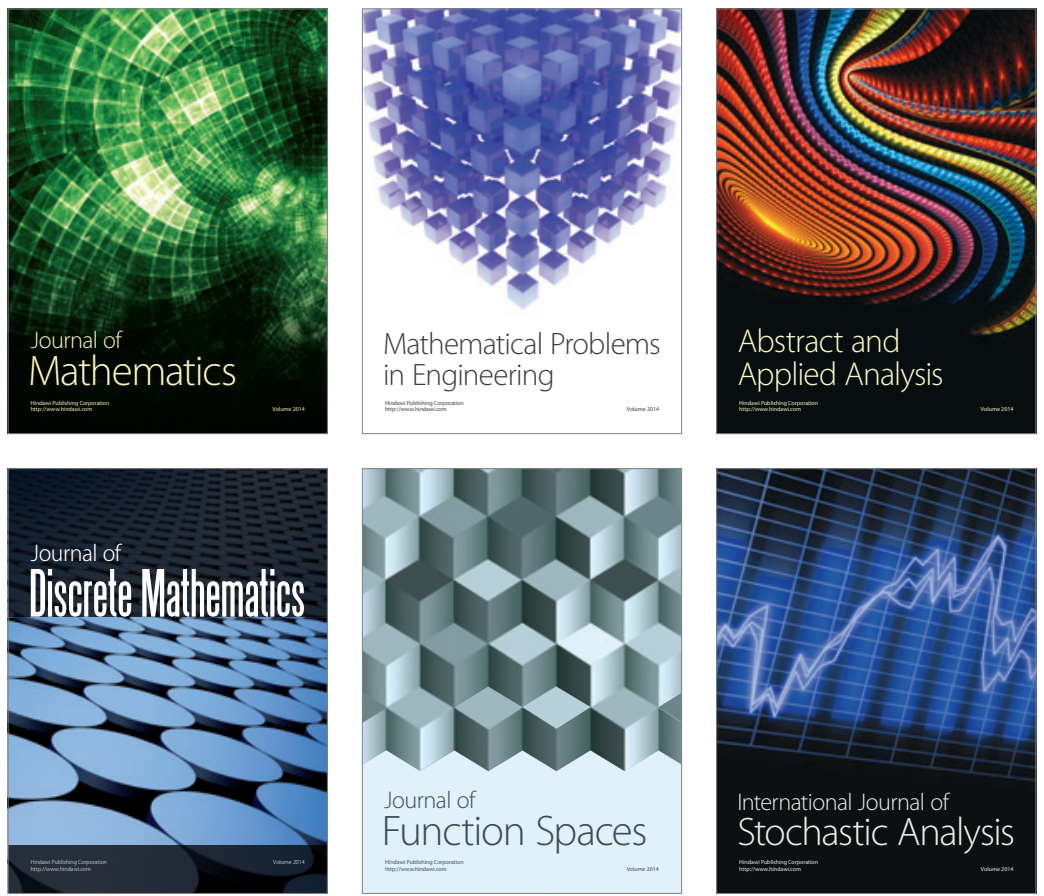

Journal of

Function Spaces



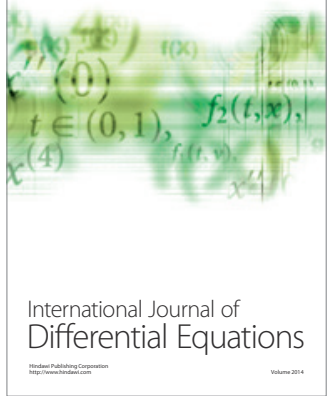
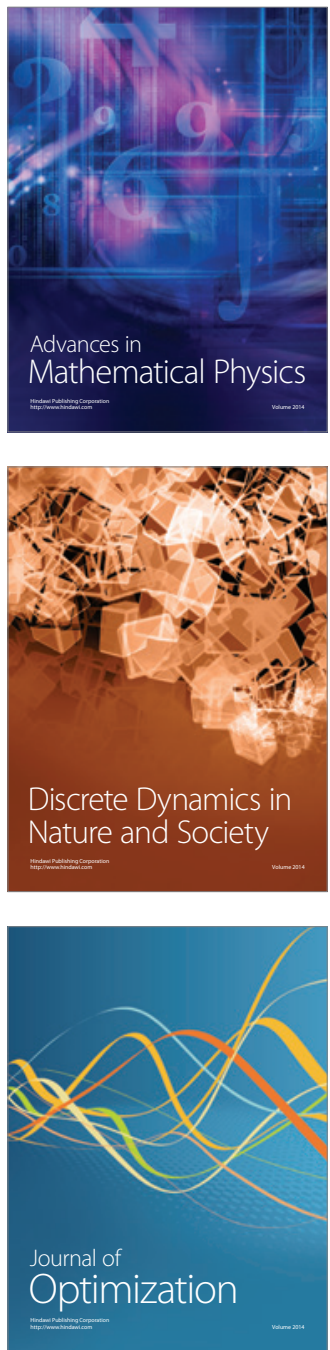